\title{
Health Related Quality of Life after Treatment of Abdominal Aortic Aneurysm with Open and Endovascular Techniques-A Two-Year Follow Up
}

\author{
Monica Pettersson ${ }^{1,2 *}$, Ingegerd Bergbom ${ }^{2}$, Erney Mattsson ${ }^{3}$ \\ ${ }^{1}$ Department of Vascular Surgery, Sahlgrenska University Hospital, Gothenburg, Sweden \\ ${ }^{2}$ Institute of Health and Care Sciences, Sahlgrenska Academy, University of Gothenburg, Gothenburg, Sweden \\ ${ }^{3}$ Department of Circulation and Medical Imaging, Norwegian University of Science and Technology, Trondheim, Norway \\ Email: *monica.e.pettersson@vgregion.se
}

Received June 20, 2012; revised July 25, 2012; accepted August 11, 2012

\begin{abstract}
Background: Patients with Abdominal Aortic Aneurysm can be treated with two different surgical methods: Open Repair (OR) or Endovascular Aortic Repair (EVAR). These two different treatments can probably result in different sense of Health Related Quality of Life, both in a short term and a long term perspective. The purpose of this prospective study was to examine patients' Health Related Quality of Life after surgical treatment of Abdominal Aortic Aneurysm over two years using different instruments for the observations. Methods: Patients were invited consecutively to answer questionnaires before operation, and 1,12 and 24 months after surgery. The study was conducted by using the Health Related Quality of Life questionnaires Short Form (SF-36) and Nottingham Health Profile (NHP). 76 patient (40 in the OR and 36 in the EVAR group) participated in the study. The mean age in the OR group were 68 years, range 52 - 80 and in the EVAR group 75 years, range 65 - 85. The results from these two groups of patients were compared to a matched reference group. Results: Both patients treated with EVAR and OR rated their Health Related Quality of Life significantly lower in the domain of Mental Health in relation to a matched reference population before surgery. This difference was not present two years after intervention. After one month Health related qualities of life were worse for the OR group. After two years significant improvements in relation to baseline were observed only among patients in the OR group. No such long-term benefits were seen in the EVAR group. Conclusions: As the component Mental Health seemed to be impaired for these study groups before surgery in relation to the matched reference group, nursing and doctors care actions may be of importance during the pre-operative phase. In the short perspective Health Related Quality of Life is worse for OR patients than the EVAR group but in the long term perspective improvements beyond preoperative status can only be seen with OR patients.
\end{abstract}

Keywords: Advanced Practice; Vascular Surgery; Health Related Quality of Life; Vascular Disease; Abdominal Aortic Aneurysm

\section{Introduction}

Patients with Abdominal Aortic Aneurysms (AAA) can be treated with Open Repair (OR) or Endovascular Aneurysm Repair (EVAR). OR is the traditional method with a higher rate of immediate cardiovascular and pulmonary complications [1,2]. The cumulative survival rates seem to be similar after two years for OR and EVAR [2]. EVAR is connected to a life-long follow up with a potential need for new interventions [3]. One can expect that these two different treatment modalities might result in different qualities of life, in a short as well as a long term perspective. In several studies Health Related Quality of Life (HRQoL) has been examined for

\footnotetext{
*Corresponding author.
}

patients treated for AAA [4-11]. Previous studies show that patients who have been subjected to OR have more pain and have a longer hospital stay than those treated with EVAR [5,7]. In a randomised study it was found that there was a small, yet significant, HRQoL advantage for EVAR as compared to OR in the early postoperative period. At 6 months and later however, patients reported better QOL after OR than after EVAR [9]. A review article have demonstrated that the results after surgery is not consistent and there are variations between benefits for OR and EVAR [12]. Using a questionnaire, Malina et al. (2000) found that about $50 \%$ of patients found OR to be a difficult experience as compared to $15 \%$ of patients treated with EVAR. No studies have simultaneously used different HRQoL instruments on the same study groups, 
to support the findings. Furthermore, no long-term studies are present. The aim of this prospective study was to examine patients' HRQoL after open and endovascular repair of AAA over two years using different instruments for the observations. It is important from a nursing perspective to investigate patients recovery process following OR and EVAR as such knowledge can be used in the preparation of patients before surgery.

\section{Theoretical Framework}

Quality of life and health is not easy to measure and Gadamer [13] stated that it is impossible to measure or quantify it. It can be described as a value related to an individual's own existence and being. The quality and meaning of life and health can change over time and thus be relative and not definite. According to Frankl [14] each human being should seek and realise certain personal meaning in life, which implies more than merely satisfying certain urges or instincts.

"Good health" is a major resource for social, economic and personal development and is an important dimension of the quality of life [15]. Health is also a phenomenon that is difficult to measure as it consist of both subjective and objective dimensions [16].

Quality of life after a specific event is assessed on the basis of one's previous life, at the time when it is measured is usually seen in relation to the quality of life that has been experienced earlier and in this way it is a summing up until the measurement takes place. Quality of life is therefore assessed from various perspectives and cannot only be made with reference to a single event, in this case an AAA operation. Although the instruments have been validated for measuring Health Related Quality of Life, it is necessary to consider a range of different aspects and events that exert an influence on the individual.

\section{Methods}

\subsection{Study Design}

An explorative quantitative design was used in which HRQoL was measured by the instruments Nottingham Health Profile (NHP) and Short Form 36 (SF-36). The combination was used since it has been indicated that SF-36 might give less skewed distribution of answers, while NHP might be more sensitive for changes in health status and changes of HRQoL over time [17]. The questionnaires were answered before operation (Baseline), 1, 12 and 24 months after surgery. A gender and age matched reference group was used for SF-36 as a comparison with the study groups. In one vascular unit the patients were invited to participate in the study when they came to the department for vascular surgery. After one month, one and two years, the questionnaires were sent to their home address. In the other vascular unit the questionnaires were sent by post at all occasions, including the preoperative evaluation.

\subsection{Instruments}

Short Form-36 (SF-36)—is a Health Related Quality of Life instrument developed by Ware \& Shelbourne (1992) to measure physical and Mental Health. The SF-36 is standardised within the framework for the quality of life assessment [18]. The instrument includes 36 items that assess eight areas of health: Physical Functioning (PF), Role limitations due to Physical health problems (RP), Bodily Pain (BP), Social Functioning (SF), Vitality (V), Mental Health (MH), Role limitations due to Emotional health problems (RE) and General Health perceptions (GH). Scores are coded for each area. The sum is calculated and transformed to a scale ranging from 0 - 100. A total score of 100 indicates the best possible state of health. The figures from these eight areas can also be summarized to either a score of the physical component (Physical Component Score, PCS) or the mental component (Mental Component Score, MCS). SF-36 has been tested for both internal and external validity as well as reliability $[19,20]$.

Nottingham Health Profile (NHP)—was developed from the earlier Nottingham Health Index [21]. The aim of the instrument is to measure the consequences in daily life of a reduced function. The instrument is divided into two main parts: Part 1 (38 questions) covers: Energy, Emotional reactions, Physical mobility, Sleep, Pain, and Social isolation. Part 2 (7 questions) covers: Paid employment, Housework, Family relationships, Social life, Sex life, Hobbies and Holiday.

The scores range from 0 - 100, with a higher score indicating a higher level of distress or impairment (opposite to SF-36). The test-retest reliability of the NHP has been investigated through correlations with a four week interval, showing a high Spearman coefficient for the separate domains [22].

\subsection{Subjects}

The inclusion criteria were: Being able to express and understand the Swedish language, mentally clear, diagnosed having AAA and acceptance to participate in the study. Totally 70 subjects were consecutively invited from a University hospital in Sweden (A) and 14 of these declined participation (6 planned for EVAR and 8 OR). The total number of included subjects from hospital A, were 56. Twenty subjects were included from a University hospital in Sweden (B) adding up to a total number of 76.

More men were included in the study ( $n=63 / 76,83 \%)$ reflecting that AAA is more common among men compared to women [23]. Baseline characteristics are described in Table 1. The mean age for all subjects was 72.5 (range 
52 - 85 years).The subjects in the OR group were significantly younger compared to the EVAR group ( $<<0.001$ ). No other significant differences in baseline characteristics were found between the two groups.

The postoperative response rates for OR and EVAR were $87.5 \%$ versus $91.7 \%$ at 1 month, $92.5 \%$ versus $83.4 \%$ at 12 month, $86 \%$ versus $90 \%$ at 24 month.

Eighty-eight percent (67/76) of the subjects could be followed until two years. In the EVAR group two patients died after one year (one each from cancer and heart disease). Two patients in the OR group died; one at one month due to heart problems and the second patient after two years of an unknown cause. The other drop-outs were related to no return of the questionnaires.

\subsection{Ethics}

All subjects were thoroughly informed about the aim of the study and their right to decline participation whenever wanted. The study was approved by the Ethical Research Committee at the Sahlgrenska Academy, University of Gothenburg, Sweden (S 712-02).

\subsection{Statistical Methods}

Mean and standard deviation (SD) were used to describe the material. Two-side p-value was used to prove significances. A p-value of $p<0.05$ was considered significant. Fisher's permutation test was used to test if there were any significant differences between the OR and EVAR groups at baseline and to calculate significant changes of variables between the two groups. Fisher's test for paired comparisons was used in order to calculate significant changes of variables over time within each group. This is equal to each group being its own control. T-test was used to see if there where any significant differences between the EVAR and OR group and the matched group at baseline and after two years. The measurements have not been performed on individual level.

Table 1. Age, gender, risk factors and p-values in the two groups: Endovascular Aneurysm Repair $(\operatorname{EVAR} \mathbf{n}=36)$ and Open Repair (OR n = 40).

\begin{tabular}{cccc}
\hline & EVAR n $=\mathbf{3 6}$ & OR n $=\mathbf{4 0}$ & p-value \\
\cline { 2 - 4 } Mean age & 75 & 68 & 0.001 \\
Range age & $65-85$ & $52-80$ & \\
Male & 32 & 31 & $>0.30$ \\
Female & 4 & 9 & 0.30 \\
Male mean age & 74 & 67 & \\
Female mean age & 78 & 72 & \\
Cerebral vascular disease & 6 & 7 & $>0.30$ \\
Cardiovascular disease & 13 & 22 & 0.19 \\
Renal disease & 4 & 7 & $>0.30$ \\
Hyper tension & 19 & 25 & $>0.30$ \\
Previous vascular operations & 7 & 15 & 0.16 \\
Pulmonary disease & 6 & 3 & $>0.30$ \\
Diabetes & 4 & 3 & $>0.30$ \\
\hline
\end{tabular}

Linear regression was used in order to investigate if any difference in age affected the comparison between the two groups. Mantels statistical test [24] was used to test if differences in pain might have affected the result. A gender and age matched reference group were randomly picked out with a two years interval from the Swedish SF-36 standard database. For the description of the matched reference group, see Swedish manual and interpretation guide [25]. The mean values for the age and gender matched reference group are described for the SF-36 domain. This reference group was only used as comparison with the study groups for the SF-36 instrument. Statistical analyses were performed using the computer program SPSS version 12.0.

\section{Results}

The hospital stay for the OR group was on average 10 days (range 5 - 17) and for the EVAR group 6 days (range 3 - 15). The aneurysmal average size before the operation was for OR $61.5 \mathrm{~mm}$ and for EVAR $61.2 \mathrm{~mm}$.

There were no significant differences in risk factors between the EVAR and OR group at baseline. There were no significant differences between the two hospitals in patient's age or risk factors. No differences emerged at baseline in the various domains of the SF-36 and the NHP with the exception of pain. The use of Mantels statistical test showed that these differences had no influence on the result. Pain and age showed no correlation. No symptoms from the aneurysm were reported by $77 \%$ $(n=57)$ of the patients.

Comparisons of OR and EVAR groups and the reference (age and gender matched) group are presented at first and thereafter differences within the groups (EVAR, $\mathrm{OR}$ ) and between the groups.

\subsection{Comparisons of OR and EVAR Groups and the Matched Reference Group (Age and Gender Matched Group)}

Baseline: The EVAR group rated their health status significantly lower in the domain of Mental Health $(\mathrm{p}<0.02)$ and MCS ( $<$ 0.002) in relation to the matched reference group. This was similar to the OR group who rated their health status significantly lower in Mental Health ( $p<0.04)$, Role Physical ( $<0.01$ ) and Role Emotional ( $<<0.03$ ).

Two years: EVAR group rated their health status similar to preoperative status except a significant improvement in the domain of Bodily Pain (BP). The OR group had a tendency to estimate their health to be improved in all domains after two years compared to the reference group but only in BP $(\mathrm{p}<0.02)$ and the Physical Component Score $(p<0.04)$ reached significance.

All mean values for EVAR and OR over time in relation to the matched reference group in the SF-36 domain are illustrated in Figures 1-8. 
Physical function

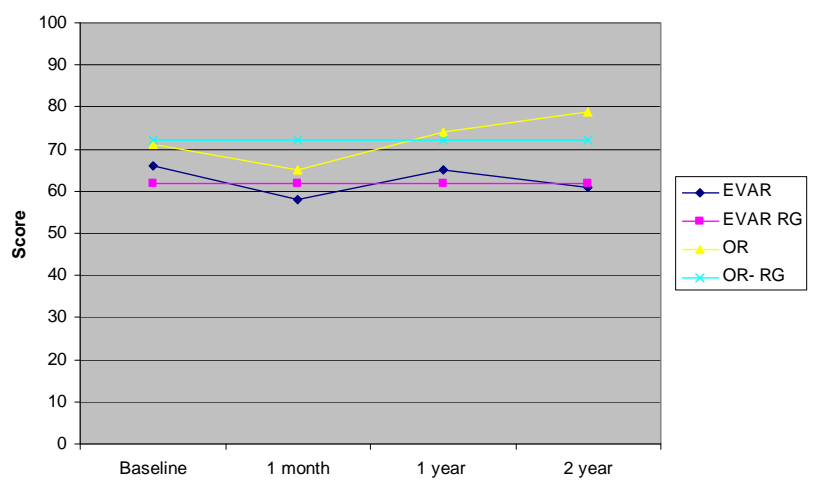

Figure 1. Changes in domains physical function (SF-36) over time for OR and EVAR patients in relation to the matched reference group (RG).

Role-physical

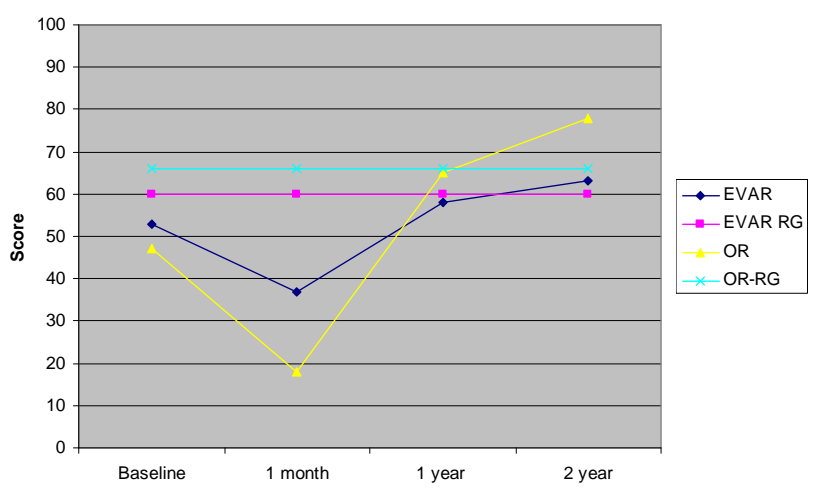

Figure 2. Changes in domains role-physical (SF-36) over time for OR and EVAR patients in relation to the matched reference group (RG).

Bodily pain

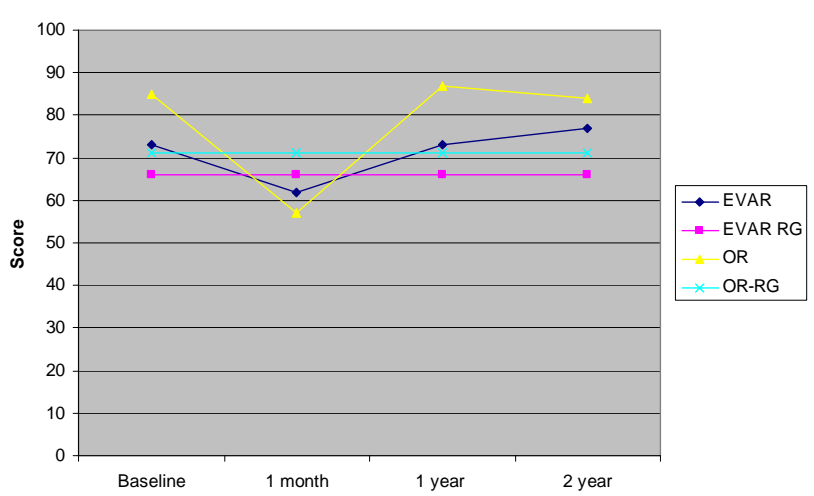

Figure 3. Changes in domains bodily pain (SF-36) over time for $O R$ and EVAR patients in relation to the matched reference group (RG).

\subsection{SF-36-Comparisons within Each Group}

After one month: A significant decrease could be seen after one month compared to baseline in both the EVAR
General health

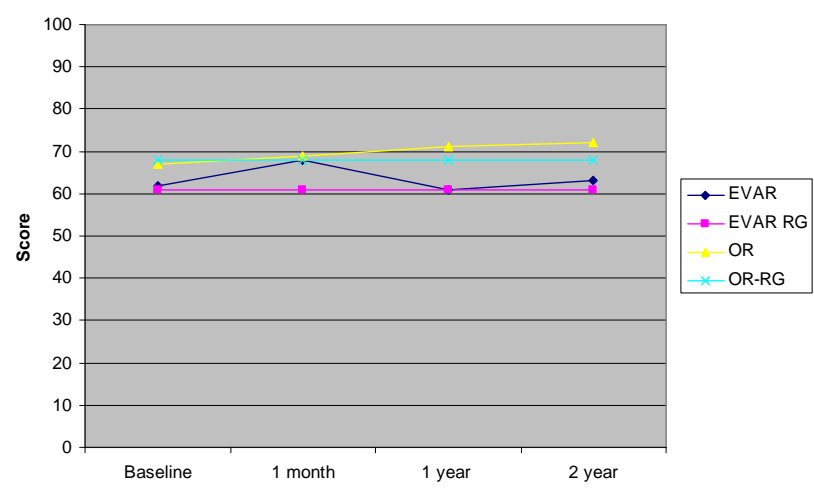

Figure 4. Changes in domains general health (SF-36) over time for OR and EVAR patients in relation to the matched reference group (RG).

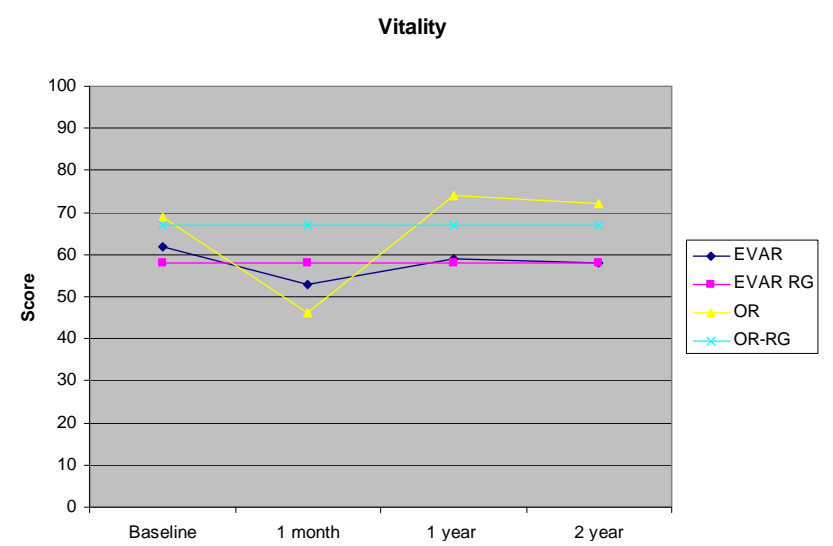

Figure 5. Changes in domains vitality (SF-36) over time for $O R$ and EVAR patients in relation to the matched reference group (RG).

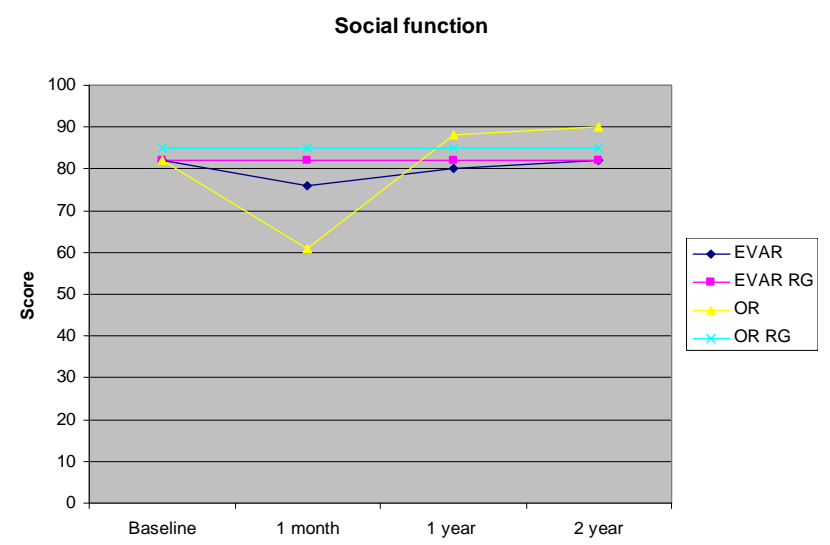

Figure 6. Changes in domains social function (SF-36) over time for OR and EVAR patients in relation to the matched reference group (RG).

and OR groups in Physical Functioning, Role Physical, Vitality and Bodily Pain. The OR group exhibited a significant decrease in the Social Functioning domain after one month, something not observed in the EVAR group. 


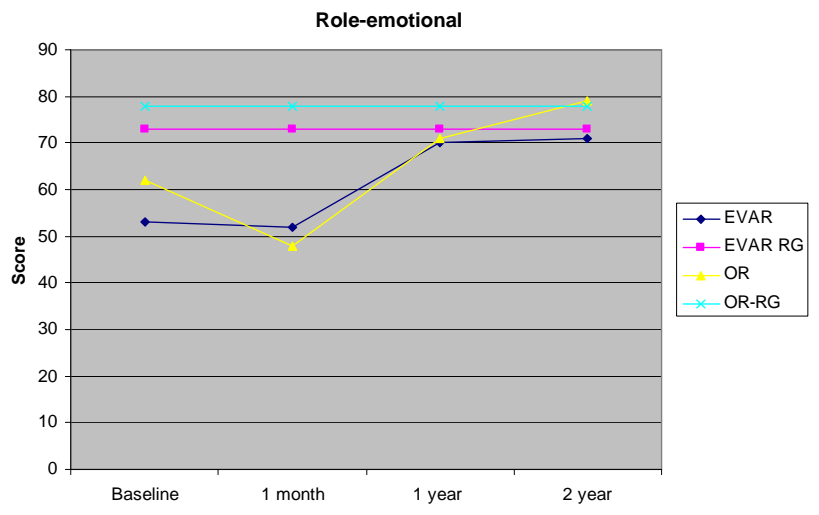

Figure 7. Changes in domains role-emotional (SF-36) over time for $O R$ and EVAR patients in relation to the matched reference group (RG).

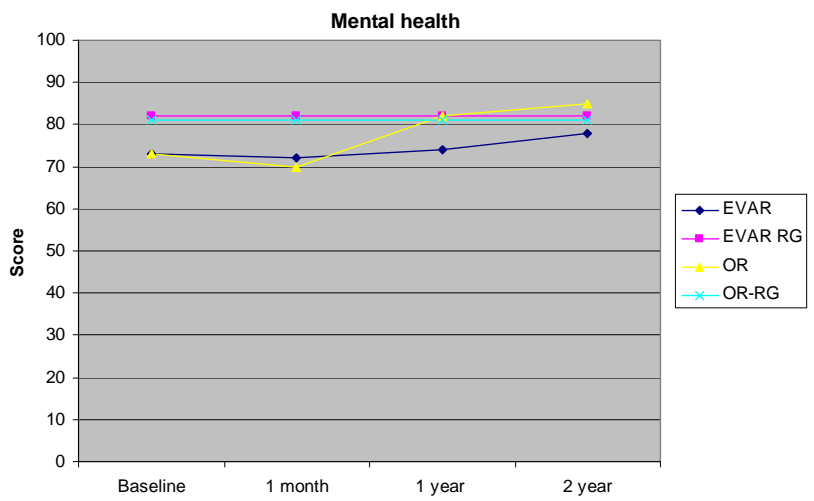

Figure 8. Changes in domains mental health (SF-36) over time for OR and EVAR patients in relation to the matched reference group (RG).
Also the general Physical Component Score decreased after one month in both groups while the Mental Component Score only decreased significantly in the OR group.

After one year: The EVAR group $(n=32)$ showed a significant improvement in the Role-emotional domain ( $p<0.022)$ compared to baseline. An improvement in the domain Mental Health $(\mathrm{n}=37$, $\mathrm{p}<0.014)$ and Rolephysical ( $\mathrm{n}=38$, $\mathrm{p}<0.025)$ was also evident in patients operated with OR after one year compared with baseline.

After two years: An improvement in the Mental Health $(\mathrm{n}=39, \mathrm{p}<0.001)$ and Role Physical $(\mathrm{n}=35, \mathrm{p}<0.001)$ was evident in patients operated with OR after two years compared with baseline. No significant differences were found in the EVAR group after two years. Physical Component Score (PCS), improved for the OR group ( $\mathrm{p}<$ $0.032)$ two years $(n=38)$ after surgery compared to baseline. No significant differences were found in the EVAR group in PCS after two years. The Mental Component Score (MCS) showed a significant improvement in the OR group from baseline until two years $(n=38$, $p<$ 0.014 ) after surgery. No significant differences were found in the EVAR group in MCS score at this time-point. Mean, SD and p-value from the scores in SF-36 over time (EVAR and OR) are presented in Tables 2 and 3.

\subsection{Comparisons between Groups over Time}

Significant differences were found between the groups at baseline and after one month concerning: Bodily Pain ( $p<0.045)$, Vitality $(p<0.020)$ and Social Functioning $(\mathrm{p}<0.037)$ with benefit for EVAR. After one year, a

Table 2. Mean, SD and p-value in domains for SF-36 for patients treated with OR (baseline, 1 month, 1 year and 2 years after surgery). (PCS = Physical Component Score, MCS = Mental Component Score). Higher scores indicate higher health related quality of life. A p-value $<\mathbf{0 . 0 5}$ compared to baseline was considered significant.

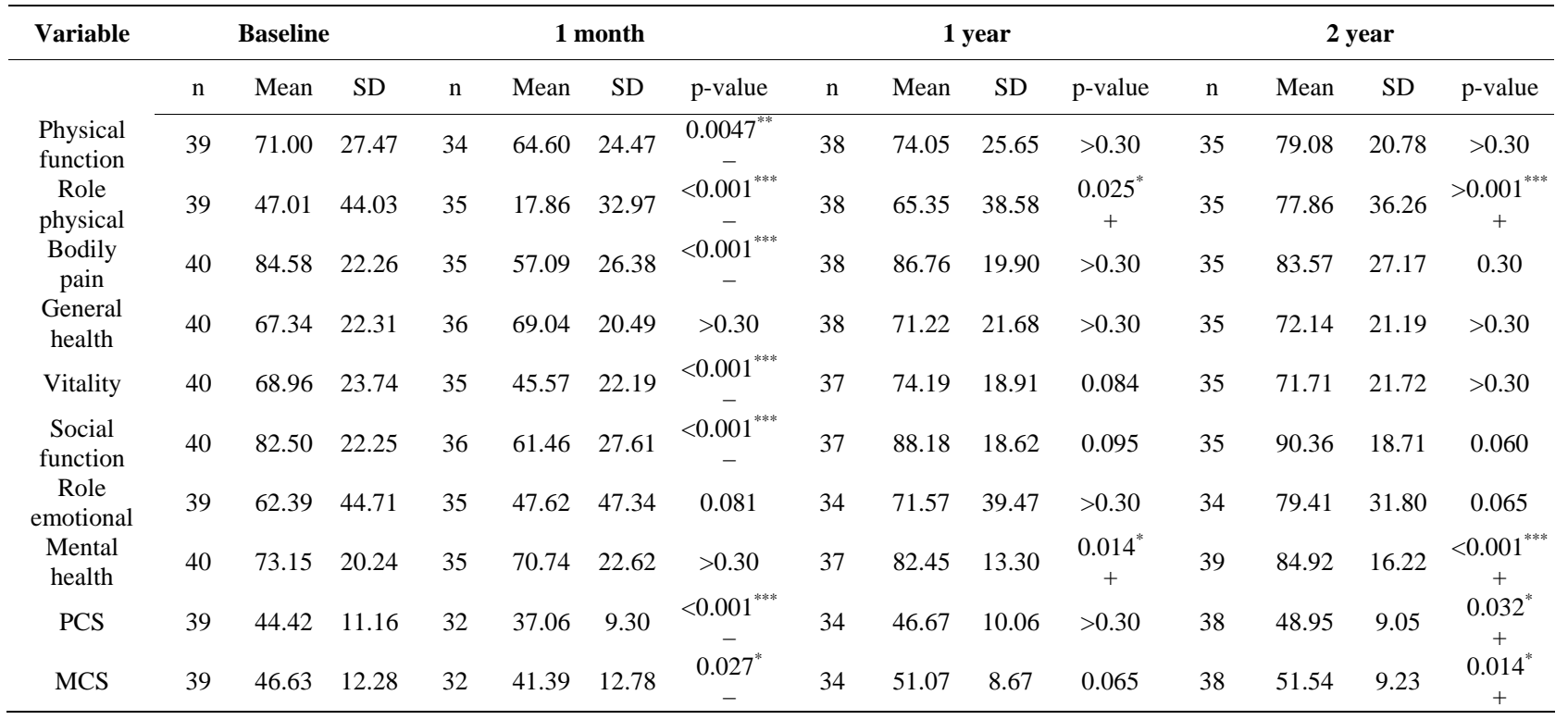

+Improvement, -Deterioration $\left(\stackrel{*}{\mathrm{p}}<0.05,{ }^{* *} \mathrm{p}<0.01,{ }^{* * *} \mathrm{p}<0.001\right)$. 
Table 3. Mean, SD and p-values in domains for SF-36 for patients treated with EVAR (baseline, 1 month, 1 year and 2 years after surgery). (PCS = Physical Component Score, MCS = Mental Component Score). Higher scores indicate higher health related quality of life. A p-value $<0.05$ compared to baseline was considered significant.

\begin{tabular}{|c|c|c|c|c|c|c|c|c|c|c|c|c|c|c|c|}
\hline \multirow[t]{2}{*}{ Variable } & \multicolumn{3}{|c|}{ Baseline } & \multicolumn{4}{|c|}{1 month } & \multicolumn{4}{|c|}{1 year } & \multicolumn{4}{|c|}{2 year } \\
\hline & $\mathrm{n}$ & Mean & SD & $\mathrm{n}$ & Mean & SD & $\mathrm{p}$-value & $\mathrm{n}$ & Mean & SD & p-value & $\mathrm{n}$ & Mean & SD & p-value \\
\hline $\begin{array}{l}\text { Physical } \\
\text { function }\end{array}$ & 36 & 65.85 & 24.81 & 33 & 57.99 & 26.00 & $\begin{array}{c}0.0025^{* *} \\
-\end{array}$ & 30 & 65.14 & 25.74 & $>0.30$ & 31 & 61.29 & 26.68 & 0.0825 \\
\hline $\begin{array}{c}\text { Role } \\
\text { physical }\end{array}$ & 36 & 52.08 & 45.66 & 33 & 37.12 & 42.45 & $\begin{array}{c}0.014^{*} \\
-\end{array}$ & 30 & 58.33 & 44.20 & $>0.30$ & 31 & 62.63 & 36.95 & 0.29 \\
\hline $\begin{array}{l}\text { Bodily } \\
\text { pain }\end{array}$ & 36 & 72.83 & 25.47 & 33 & 62.42 & 27.37 & $\begin{array}{c}0.020^{*} \\
-\end{array}$ & 31 & 73.35 & 28.72 & $>0.30$ & 31 & 77.23 & 26.50 & $>0.30$ \\
\hline $\begin{array}{l}\text { General } \\
\text { health }\end{array}$ & 35 & 62.14 & 21.81 & 33 & 67.61 & 20.81 & $>0.30$ & 31 & 60.89 & 21.47 & 0.18 & 31 & 63.51 & 17.98 & $>0.30$ \\
\hline Vitality & 35 & 61.57 & 22.18 & 32 & 52.97 & 21.25 & $\begin{array}{c}0.0098^{* *} \\
-\end{array}$ & 31 & 59.03 & 23.32 & 0.096 & 31 & 57.90 & 22.24 & 0.092 \\
\hline $\begin{array}{c}\text { Social } \\
\text { function }\end{array}$ & 36 & 81.94 & 19.70 & 33 & 76.52 & 23.12 & 0.089 & 31 & 79.44 & 29.15 & $>0.30$ & 31 & 81.85 & 25.17 & $>0.30$ \\
\hline $\begin{array}{c}\text { Role } \\
\text { emotional }\end{array}$ & 34 & 52.94 & 45.78 & 32 & 52.08 & 44.75 & $>0.30$ & 31 & 70.43 & 42.10 & $\begin{array}{c}0.022^{*} \\
+\end{array}$ & 31 & 70.97 & 38.24 & 0.068 \\
\hline $\begin{array}{l}\text { Mental } \\
\text { health }\end{array}$ & 35 & 72.69 & 19.75 & 32 & 72.41 & 20.89 & $>0.30$ & 31 & 74.32 & 19.67 & $>0.30$ & 36 & 78.36 & 19.43 & 0.12 \\
\hline PCS & 32 & 41.63 & 10.96 & 31 & 37.99 & 10.92 & $\begin{array}{c}0.0037^{* *} \\
-\end{array}$ & 30 & 41.33 & 11.97 & 0.077 & 36 & 40.05 & 13.39 & 0.062 \\
\hline MCS & 32 & 45.79 & 10.51 & 31 & 45.42 & 12.22 & $>0.30$ & 30 & 48.35 & 12.81 & 0.29 & 36 & 49.82 & 11.61 & 0.055 \\
\hline
\end{tabular}

+Improvement, -Deterioration $\left({ }^{*} \mathrm{p}<0.05,{ }^{* *} \mathrm{p}<0.01\right)$.

significant difference in Vitality $(\mathrm{p}<0.015)$ was revealed, to the advantage of the OR group, while after two years a benefit was revealed in the Physical Component Score domain $(\mathrm{p}<0.0056)$ for patients operated with OR, Table 4.

\subsection{NHP-Comparison within Each Group}

After one month: Significant differences in relation to baseline in the health status of the OR group were found after one month in the following domains; Lack of energy $\mathrm{p}<0.011)$, Pain $(\mathrm{n}=36, \mathrm{p}<0.001)$, Physical mobility ( $\mathrm{n}=36, \mathrm{p}<0.001$ ), which was not observed in the EVAR group. Overall NHP increased significantly in patients operated on by means of OR $(n=36, p<0.017)$ after one month, meaning reduced HRQoL. No significant differences were found in the EVAR group.

After one year: No significant differences were found in the EVAR and OR groups between baseline and observations at one year. The result indicates that the OR-group had recovered from the intervention.

After two year: The EVAR group reported a decline in health status after two years in the domains Social isolation ( $\mathrm{n}=31), \mathrm{p}<0.027$ ) and Physical mobility ( $\mathrm{n}=31$, $\mathrm{p}$ $<0.020$ ). The domain of sleep improved in the EVAR group compared to baseline. No significant differences were found in the OR group compared to baseline values.

\subsection{Comparison between the Groups over Time in NHP}

After one month most domains demonstrated a significant change to baseline with OR which was not the case with EVAR. Significant differences between the groups were identified: after 1 month in the domain of Pain $(p<0.028)$ with benefit for patients operated with EVAR. After 2 years a significant difference in sleep $(<0.015)$ between EVAR and OR was found in favour of patient operated with EVAR. The total NHP-score was however in favour of OR after 1 and 2 years, especially demonstrated with pain, social isolation and physical mobility, Table 5.

\section{Discussion}

Aortic aneurysm is a serious diagnosis that may affect patients' whole life. This study shows that Mental Health is worse for patients with diagnosed AAA in relation to an age and gender matched population. Two years after surgery patients have similar scores as the matched reference group, and within the domain of Physical Component Score, patients who were operated with OR reported even better health. Consequently surgery for AAA must be considered as a benefit from a HRQoL perspective. HRQoL should therefore be considered to be part of the decision making besides aneurysmal diameter. The average age in this study emerged from the consecutive inclusion. It is possible that the results of this study would have been different if it had been a younger population. In this study, however, each group was also its own reference when comparisons were made. Dick et al. (ref nr) showed, in a study with a larger number of subjects (compared to our study) that long-term HQoL measured by the SF-36 after EVAR and OR, that scores in all age groups were similar to an age-and sex-adjusted standard population. 
Table 4. Fischer's permutation test for pair significant comparisons between the two groups OR and EVAR (SF-36) (p-values between the group). From baseline to 1 month, 1 and 2 year after surgery. In the domain of bodily pain, vitality and social function it was a benefit for the EVAR group after 1 month. After 1 year, a significant difference in vitality was revealed, to the advantage of the OR group, while after 2 years a benefit was revealed in the physical component score domain for patients operated with OR.

\begin{tabular}{cccc}
\hline Variable & 1 month & 1 year & 2 year \\
\hline Psychical function & $>0.30$ & $>0.30$ & 0.12 \\
Role-psychical & $>0.30$ & $>0.18$ & 0.16 \\
Bodily pain & $0.045^{*}$ & $>0.30$ & $>0.30$ \\
General Health & $>0.30$ & 0.20 & $>0.30$ \\
Vitality & $0.020^{*}$ & $0.015^{*}$ & $>0.30$ \\
Social function & $0.037^{*}$ & 0.094 & $>0.11$ \\
Role-emotional & $>0.30$ & 0.021 & $>0.30$ \\
Mental-health & $>0.30$ & $>0.18$ & 0.29 \\
PCS & 0.18 & 0.13 & $0.0056^{*}$ \\
MCS & 0.25 & $>0.30$ & $>0.30$ \\
\hline
\end{tabular}

Table 5. Fischer's permutation test for significant comparison between the two groups EVAR and OR (NHP) (p-values between the groups). From baseline to 1 month, 1 and 2 year after surgery. In the domain of pain after 1 month and sleep after 2 years it was a benefit for the EVAR group.

\begin{tabular}{cccc}
\hline Variable & 1 month after & 1 year after & 2 year after \\
\hline Emotional & 0.27 & $>0.30$ & $>0.30$ \\
Sleep & $>0.30$ & $>0.30$ & $0.015^{*}$ \\
Energy & 0.21 & 0.15 & $>0.30$ \\
Pain & $0.028^{*}$ & $>0.30$ & 0.21 \\
PM & 0.093 & $>0.30$ & 0.17 \\
Social isolation & $>0.30$ & $>0.30$ & $>0.30$ \\
NHP total & 0.18 & $>0.30$ & $>0.30$ \\
NHP 2 & 0.30 & $>0.30$ & $>0.30$ \\
\hline
\end{tabular}

This prospective study had a high response frequency and few cases lost for follow-up. Some of the patients in the study had foreign backgrounds but since they could speak and understand the Swedish language, we have not identified them from the rest of the group. It is a weakness that patients with different origins and who not can speak and understand the Swedish language not are represented in this study when we have an increasingly multicultural society and this is something to consider in future studies. The study indicates that patients in the EVAR group reported a better HRQoL in the short time perspective when compared to OR patients but, after one and two years after the intervention, the measurements (SF-36) revealed that the OR group had an improved HRQoL in several domains, which was not the case with EVAR. These findings were strengthened by the NHP measurement where the EVAR group showed a decline in HRQoL after two years in the areas of Social isolation (SO) and Physical mobility (PM), which was not evident in the OR group. The finding that patients in the EVAR group reported impaired HRQoL two years after the intervention could be explained by the fact that annual medical follow-ups may keep patients in an uncertain position, reminding them of the vulnerability of life and the intervention.

Our study revealed that OR patients exhibited a significant improvement in the Mental Health domain after one and two years, which was not the case with the EVAR patients and thus agrees with the findings of Aljabri et al. 2006.

Several studies have shown that shortly after the operation OR patients have a poorer HRQoL in several domains compared to EVAR patients [4-10]. In the present study overall NHP showed a significant decreased in HRQoL in the OR groups but not in EVAR group at one month. As mentioned a different situation seems to be present in the long term perspective. In a randomized study by Prinssen et al. 2004 [9], which included 153 patients, it was found that OR was associated with a significantly higher HRQoL at one year following treatment in the domains of Social function (SF), Role Emotional (RE) and Mental Health. This result is in accordance with findings in our study, which also showed an improvement in Mental Health from baseline to one and two years after surgery for patients treated with OR.

One HRQoL aspect which is not specifically covered in this study is the impact on sexual function. It has previously shown that this part of the HRQoL is affected similarly with a significant deteriorated function over time with EVAR but not OR [26]. EVAR-1 stated that no differences in HRQoL had been observed among patients after 1 - 24 months. This study showed that EQ5D (EuroQol) scores were similar in both groups and to agematched and sex-matched population norms. At $12-24$ months after randomization there was no difference between the groups. HRQoL were secondary endpoints in this study.

The results in EVAR-1 reported only MCS and PCS and not specific for the different domains in SF-36. There were no final observations of the Health Related Quality of Life two years after surgery. Point of times for reporting were, $0-3,3-12$ and 12 - 24 months which unfortunately gives a potential of spread of the responses over time. HRQoL differs over time, as shown in the present study, which might have influenced the conclusions in EVAR-1.

In our study patients where not randomly assigned for OR or EVAR. Instead the respondents were invited consecutively. Patients might have been operated with EVAR due to the fact that their medical condition was too poor for an OR. However, risk factors and characteristics did not differ between the groups.

The interviews with the patients revealed that their health 
status is affected by many other conditions that are not related to the aneurysm. On the other hand the additional questions, which can be considered more disease specific, revealed that the majority of OR patients experienced the operations as a difficult event. This agrees with the Health Related Quality of Life instruments that indicated a greater decrease in the perception of health a short time after the OR compared to the patients in the EVAR group.

There are some limitations that must be considered when discussing the results. There was a significant difference in age in our study. The patients operated with EVAR were older than the OR group, which obviously can have consequences for the results. However, this fact was taken into account when performing the statistical analyses and each group has been its own control. The relative change within each group has been used for comparisons between EVAR and OR. In another study where the EVAR group also were older than their OR counterparts the EVAR group reported a poorer health quality of life six months after the operation compared to the OR patients [10].

Another weakness of this study is that we have not taken into account the subjects' social network and cognitive status, and we have not investigated the subjects' asset of supportive next of kin. Such factors may have an impact on the perceived Health Related Quality of Life.

HRQoL after a specific event is assessed on the basis of one's previous life, as this is the only way in which the present life situation can be evaluated. Although the instruments have been validated for measuring quality of life, it is necessary to consider a range of different aspects and events that exert an influence on the individual and responses to questionnaires. Therefore caring in conjunction with prospective follow ups should bring these issues concerning health and quality of life up and in that way alleviate worries and other discomforts. This presupposes of course an establishment of a caring relationship [27].

The conclusions we have drawn are based on a small number of subjects, however the number of subjects included in the study are similar to previously published studies [4-10].

\section{Conclusions}

Mental Health seemed to be impaired in AAA patients in relation to a matched reference group. This difference is not present two years after intervention.

In the short term (one month) perspective, HRQoL seems to be worse for the OR group than the EVAR group. In the long term perspective however (one and two years), there were significant improvements compared to preoperatively values in the OR group in the domains; Rolephysical and Mental Health and in the Physical Component Score (PCS) and the Mental Component Score (SF-36), which was not seen with EVAR. The NHPscore was also in favour for OR after one and two years.
In summary the mental quality of life improves for AAA patients independent of treatment modality. In the short time perspective OR is worse than EVAR. After one and two years the patients subjected to OR have better HRQoL than EVAR patients compared to their baseline values. The OR patients also seem to mentally include their treatment in the general life to a higher degree at later stages.

\section{Relevance to Clinical Practice}

Consequences of OR seem to affect physical well-being in another way for OR patients than for patients where the EVAR method was used. The findings from this study are important for further development of the care plans. Time for conversation about patient's experiences, questions and thoughts during the process, but also in the preoperative phase is important for preventing a decrease in Health Related Quality of Life both before and after surgery.

\section{Acknowledgements}

The Vascular Surgery in west Sweden and Odd Fellow Logen 129, Klarälven, Sweden supported the study. The authors are grateful to the patients for participation in the study.

\section{REFERENCES}

[1] J. D. Blankensteijn, et al., "Dutch Randomized Endovasculär Aneurysm Manegment (Dream Trial Group), Two Year Outcomes after Conventional or Endovascular Repair of Abdominal Aortic Aneurysm," New England Journal Medicine, Vol. 23, No. 352, 2005, pp. 352-405.

[2] EVAR 1 Trial Participants, "Endovascular Aneurysm Repair versus Open Repair in Patients with Abdominal Aortic Aneurysm (EVAR Trial 1): Randomised Controlled Trial," Lancet, Vol. 365, No. 9478, 2005, pp. 2179-2186. doi:10.1016/S0140-6736(05)66627-5

[3] R. M. Sandfjord, et al., "Endovascular Abdominal Aortic Aneurysm Repair: 5-Year Follow-Up Results,” Annals of Vascular Surgery, Vol. 3, No. 22, 2008, pp. 372-378.

[4] A. J. Lloyd, et al., "Comparison of Cognitive Function and Quality of Life after Endovascular or Conventional Aortic Aneurysm Repair,” British Journal of Surgery, Vol. 87, No. 4, 2000, pp. 443-447. doi:10.1046/j.1365-2168.2000.01428.x

[5] M. Malina, et al., "Quality of Life before and after Endovascular and Open Repair of Asymptomatic AAAs: A Prospective Study," Journal of Endovascular Therapy, Vol. 7, No. 7, 2000, pp. 372-379. doi:10.1583/1545-1550(2000)007<0372:QOLBAA>2.0.C $\underline{\mathrm{O} ; 2}$

[6] J. L. Ballard, et al., "Quality of Life before and after Endovascular and Retroperitoneal Abdominal Aortic Aneurysm Repair," Journal of Vascular Surgery, Vol. 4, No. 
39, 2001, pp. 797-803.

[7] R. V. Aquino, et al., "Quality of Life Assessment in Patients Undergoing Endovascular or Conventional AAA Repair," Journal of Endovascular Therapy, Vol. 8, No. 58, 2001, pp. 521-528. doi:10.1583/1545-1550(2001)008<0521:QOLAIP >2.0.C $\underline{\mathrm{O} ; 2}$

[8] P. E. Lottman, et al., "Health-Related Quality of Life Outcomes Following Elective Open Repair or Endovascular AAA Repair," Journal of Endovascular Therapy, Vol. 11, No. 3, 2004, pp. 23-29. doi:10.1583/03-1141.1

[9] M. Prinssen, et al., "Quality of Life after Endovascular and Open AAA Repair. Results of a Randomised Trial,” Journal of Vascular Surgery, Vol. 27, No. 2, 2004, pp. 121-127.

[10] B. Aljabri, et al., "Patient-Reported Quality of Life after Abdominal Aortic Aneurysm Surgery: A Prospective Comparison of Endovascular and Open Repair,” Journal of Vascular Surgery, Vol. 44, No. 6, 2006, pp. 1182-1187. doi:10.1016/j.jvs.2006.08.015

[11] F. Dick, et al., "Outcome and Quality of Life in Patients Treated for Abdominal Aortic Aneurysms: A Single Center Experience," World Journal of Surgery, Vol. 6, No. 6, 2008, pp. 987-994. doi:10.1007/s00268-008-9565-1

[12] N. Muszbek, et al., "Systematic Review of Utilities in Abdominal Aortic Aneurysm," European Journal of Vascular and Endovascular Surgery, Vol. 3, No. 36, 2008, pp. 283-289. doi:10.1016/j.ejvs.2008.03.018

[13] H.-G. Gadamer, "Truth and Method," Continuum, New York, 1989.

[14] V. Frankl, "Livet Måste ha Mening (Mans Search for Meaning),” Natur \& Kultur, Stockholm, 1986.

[15] World Health Organisation, "WHOQOL Study Protocol: The Development of the World Health Organisation Quality of Life Assessment Instrument: Publication MNH/ PSF/93.9 Geneva, Switzerland: Division of Mental Health,” World Health Organization, 1993.

[16] K. Eriksson, "The Theory of Caritative Caring: A Vision" Nursing Science Quarterly, Vol. 3, No. 20, 2007, pp. 201-202. doi:10.1177/0894318407303434

[17] R. Klevsgård, et al., "Nottingham Health Profile and Short-Form 36 Health Survey Questionnaires in Patients with Chronic Lower Limb Ischemia: Before and after
Revascularization,” Journal of Vascular Surgery, Vol. 2, No. 36, 2002, pp. 310-317.

[18] J. E. Ware and C. D. Shelbourne, "The MOS 36-Item Short-Form Health Survey (SF-36) Conceptual Framework and Item Selection,” Medicine Care, Vol. 30, No. 6, 1992, pp. 473-484. doi:10.1097/00005650-199206000-00002

[19] J. E. Brazier, et al., "Validating the SF-36 Health Survey Questionnaire: New Outcome Measure for Primary Care,” British Medical Journal, Vol. 6846, No. 305, 1992, pp. 160-164.

[20] WHO-QOL, "Study Protocol for the World Health Organisation Project to Develop Quality of Life Assessment Instrument," Quality of Life Research, Vol. 2, No. 2, 1993, pp. 153-159. doi:10.1007/BF00435734

[21] S. M. Hunt and J. McEwen, "The Development of a Subjective Indication,” Sociol Health Illness, Vol. 3, No. 2, 1980, pp. 231-246. doi:10.1111/1467-9566.ep11340686

[22] S. M. Hunt, et al., "Reliability of a Population Survey Tool for Measuring Perceived Health Problems: A Study of Patients with Osteoarthrosis,” Epidemiology Community Health, Vol. 35, No. 4, 1981, pp. 185-188. doi:10.1136/jech.35.4.297

[23] B. G. Derubertis, et al., “Abdominal Aortic Aneurysm in Women: Prevalence, Risk Factors, and Implications for Screening,” Journal of Vascular Surgery, Vol. 4, No. 46, 2007, pp. 630-635. doi:10.1016/j.jvs.2007.06.024

[24] N. Mantel, "Chi-Square Tests with One Degree of Freedom; Extension of the Mantel-Haenszel Procedure,” Journal of the American Statistical Association, Vol. 58, No. 303, 1963, pp. 690-700. doi:10.2307/2282717

[25] M. Sullivan and C. Taft, "SF-36 Health Survey Manual and Interpretation Guide 2: Edition,” Göteborg University, Gothenburg, 2002.

[26] M. Pettersson, et al., "Prospective Follow-Up of Sexual Function after Elective Repair of Abdominal Aortic Aneurysm Using Open and Endovascular Techniques,” Journal of Vascular Surgery, Vol. 50, No. 3, 2009, pp. 492-499. doi:10.1016/j.jvs.2009.02.245

[27] L. Berg, et al., "Caring Relationship in a Context-FieldWork in a Medical Ward," International Journal of Nursing Practice, Vol. 3, No. 12, 2007, pp. 100-106. 